\title{
Die Liste, paradigmatisch
}

\author{
Matthias Schaffrick • Niels Werber
}

Online publiziert: 13. September 2017

(C) Springer-Verlag GmbH Deutschland 2017

\section{Die Kontingenz der Liste}

Listen werden zumeist unterschätzt, weil sie uns so einfach und selbstverständlich vorkommen. Sie begegnen uns jeden Tag in allen möglichen Situationen, und unser inniges und vertrautes, alltägliches Verhältnis zur Liste macht uns blind für die Prozesse, die beim Erstellen, Verwalten und Gebrauchen einer Liste ablaufen. ${ }^{1}$ Schaut man etwas genauer hin, stellen sich Listen anders dar.

Jack Goody schreibt in einem zum Klassiker der Listenforschung avancierten Text von 1977, »What's in a List?«: »Die Art, wie Wörter (oder >Dinge <) in einer Liste angeordnet werden, ist selbst eine Art der Klassifikation oder der Eingrenzung eines >semantischen< Feldes, denn der Vorgang impliziert, daß einzelne Elemente ein- und andere ausgeschlossen werden « (Goody 2012, S. 384). In diesem Text

\footnotetext{
1 Bemerkenswert ist, dass auch Texte über Listen in der Regel mit Listen beginnen. (Man möge diese Beobachtung an den Texten dieser Ausgabe der Zeitschrift für Literaturwissenschaft und Linguistik überprüfen.) Es wird aufgelistet, was man alles mit Listen machen kann, wo überall sie uns begegnen und wie sie unsere Handlungsweisen mitbestimmen. Das ist insofern bemerkenswert, als literaturwissenschaftliche Aufsätze über Romane in der Regel nicht damit anfangen, einen Roman zu erzählen, oder Abhandlungen über Gedichte meist nicht in gebundener Sprache abgefasst werden. Obwohl Listen über die wissenschaftlichen Disziplinen hinweg zu einem viel beachteten Gegenstand geworden sind, bleibt die Beschäftigung mit Listen aufgrund der auf den ersten Blick offensichtlichen Eigenschaften von Listen - ihrer alläglichen Selbstverständlichkeit und formalen Einfachheit - wohl rechenschaftspflichtig. Diese Rechtfertigung erfolgt zumeist dadurch, das Funktionsspektrum oder die Ubiquität von Listen durch eine Auflistung zu veranschaulichen. Damit aber erfolgt die Rechtfertigung des Themas selbst in der Form des zu Rechtfertigenden, nämlich als Liste. Den Praktiken des Auflistens ist offenbar nicht zu entkommen.
}

M. Schaffrick $(\square) \cdot$ N. Werber

Germanistisches Seminar, Universität Siegen, Siegen, Deutschland

E-Mail: schaffrick@germanistik.uni-siegen.de

N. Werber

E-Mail: werber@germanistik.uni-siegen.de 
nimmt Goody die beim Umgang mit Listen ablaufenden Prozesse der De- und Rekontextualisierung, der Transformation und Modifikation, der Inklusion und Exklusion in den Blick. Er konstatiert eine performative, epistemologische Kraft der Liste, die von der Art und Weise abhänge, wie die Elemente (Wörter, Dinge) auf einer Liste angeordnet werden. Die verschiedenen Möglichkeiten der Anordnung hängen davon $a b$, in welcher Reihenfolge die Elemente gebracht werden; ob sie durchnummeriert, also aufgezählt; ob sie nominal, also lediglich genannt; ob sie alphabetisch aufgelistet werden; ob sie untereinander oder nebeneinander geschrieben werden. Überhaupt schafft erst die Verschriftlichung der Elemente auf der Liste diejenige Ordnung des Wissens, die ohne die Liste so nicht denkbar gewesen wäre. Die schriftmediale Ordnung der Liste bildet also die Bedingung der Möglichkeit für Formation und Vermittlung von Wissensbeständen. Das Auflisten selbst ist eine seit den sumerischen Anfängen der Schrift tradierte epistemische Praktik in dem Sinne, dass die Liste die auf ihr versammelten Elemente klassifiziert oder eine Isotopie aufbaut, indem sie Wörter mit gleichen semantischen Merkmalen zusammenführt. Den semantischen und epistemischen Ordnungen liegt aber nicht nur die Entscheidung darüber zugrunde, wie etwas auf der Liste angeordnet wird, sondern auch was überhaupt auf die Liste gesetzt wird (vgl. ebd., S. 389), welche Elemente ein- und welche ausgeschlossen werden. Das Aufgelistete steht also im Horizont all derjenigen Elemente, die nicht auf der Liste stehen, dort aber potenziell einen Platz hätten haben können. Daher führt jede Liste die »Notwendigkeit von Grenzziehungen mit sich« (ebd., S. 387), die stets auch anders ausfallen könnten.

Listen sollten also keinesfalls unterschätzt werden, denn vor dem hier skizzierten Hintergrund wird ersichtlich, wie wenig einfach, sondern höchst komplex, und wie kontingent und damit alles andere als selbstverständlich die Ordnung der Liste ist. Wenn wir Listen schreiben oder uns bei unseren Handlungen oder Entscheidungen an Listen orientieren, müssen wir uns über die Kontingenz der Liste keine Gedanken machen. Wenn man aber die bei Goody genannten Eigenschaften und Effekte der Liste bedenkt, scheinen Listen, ihre Formen und Verfahren sowie ihre ordnungsstiftenden und handlungsleitenden Effekte literatur-, kultur- und medienwissenschaftliche ebenso wie soziologische Analysen geradezu herauszufordern. Die Rede von >Klassifikation < und von >Eingrenzung <, von >semantischem Feld < und von >ein- und ausschließen < suggeriert jedenfalls, dass Listen sprachlich und medial konstruierte Formen sind, die soziale und epistemische Ordnungen konstituieren. Die Verfahren, die bei der Erstellung von Listen ablaufen, sind die Selektion und die darauf folgende Kombination der Elemente, und diese Verfahren wiederum werden als Praktiken des Ein- und Ausschließens, der Eingrenzung und Klassifikation sozial wirksam.

Listen wirken abgeschlossen und vollständig, während sie in den meisten Fällen erweiterbar oder ihre Einträge durch andere Elemente substituierbar sind. Im Hinblick auf diese kontingente Form der Liste ist es bemerkenswert, dass Goody die »Art, wie Wörter (oder >Dinge <) in einer Liste angeordnet werden«, gegenüber dem, was eigentlich angeordnet wird, betont. Die Kontingenz der Liste ergibt sich nicht allein daraus, welche Elemente auf der Liste stehen, sondern aus der Form, in der sie angeordnet werden. Was von narratologischer Warte aus bei der Analyse von Erzählungen entscheidend ist, gilt laut Goody auch bei der Analyse von Listen. Besondere Bedeutung liegt in der Erzähltheorie nämlich auf dem discours, also der Art 
und Weise, wie etwas erzählt wird, im Gegensatz zur histoire, also der Geschichte, von der erzählt wird, dem »Was« der Erzählung. »Jede Geschichte lässt sich auf verschiedene Weise erzählen«, heißt der Grundsatz der Erzähltheorie (Martínez/ Scheffel 2012, S. 29). Jede Liste lässt sich auf verschiedene Weise ordnen, müsste der Kontingenzgrundsatz einer Theorie der Liste lauten.

\section{Listen sind keine Narrative}

Die Kontingenz des Erzählens und Auflistens ergibt sich aus der Vielzahl möglicher Erzähl- oder Auflistungsverfahren, also daraus, dass sich etwas auf ganz verschiedene Weise erzählen beziehungsweise auflisten lässt. Die Verfahren des Erzählens und Auflistens unterscheiden sich jedoch signifikant voneinander. Listen zeichnen sich gerade dadurch aus, dass sie nicht erzählen, sondern »the limits of narration« markieren, »which are inherent in the list as an element that defies the sequentiality of narrative and breaks open the successive flow of events « (Contzen 2016, S. 241). Listen liegen im Grenzbereich des Narrativen. Zuweilen regen sie als »Text gelesen« dazu an, zu erzählen (Mainberger 2003, S. 31), oder sie pendeln - wie bei Georges Perec - zwischen Aufzählung und Erzählung ironisch hin und her (vgl. ebd., S. 33), sodass man sagen könnte, Listen seien pränarrativ, insofern sie den »Kern eines potenziellen Narrativs « enthalten (ebd, S. 31). ${ }^{2}$ Dennoch sind Listen per definitionem keine Narrative. ${ }^{3}$

Das Narrative hat sich in den vergangenen dreißig Jahren, also nachdem Lyotards »Ende der Großen Erzählungen« selbst als »Große Erzählung « enttarnt war, als ein dominantes Paradigma der Literatur- und Kulturwissenschaften etabliert (vgl. Koschorke 2004, 2012; Müller-Funk 2008; Meuter 2011; Nünning 2013) - und zwar derart dominant, dass man sich fragte, welche anderen kulturwissenschaftlichen Paradigmen es damit aufnehmen könnten. Neuerdings, das heißt seit etwa zehn Jahren, jedoch findet das anti-narrative Paradigma der Liste vermehrt literatur- und kulturwissenschaftliche sowie soziologische Aufmerksamkeit, wodurch die Dominanz des Narrativen infrage gestellt wird.

Weder im Sinne antiker aristotelischer Poetik sind Listen Erzählungen, d.h. ein Ganzes, »was Anfang, Mitte und Ende hat« (Poetik, 7), noch im Sinne neuerer

\footnotetext{
${ }^{2}$ Vgl. zu Perecs literarischen >Ethnologie der eigenen Kultur< den Beitrag von Sabine Mainberger in diesem Heft.

${ }^{3}$ Der nicht-narrative Charakter der Liste ist common sense unter den einschlägigen Beiträgen zur Listenforschung. Eva von Contzen definiert Listen als »inherently nonnarrative elements « (Contzen 2016, S. 245). Auch Urs Stäheli konstatiert, dass »the list has no narrative structure (Stäheli 2012b, S. 236), sie verfüge über »no narrative that would produce a meaningful and causal chain of events« (ebd., S. 238). Ebenso argumentieren Marieke Goede, Anna Leander und Gavin Sullivan in ihrer Einleitung zum Environment and Planning $D$ Themenheft The politics of the list (2016): »What makes the list distinct is its non-narrative form [...]. Lists procedure categories by making a >cut in the continuous flow of the world « (de Goede/Leander/Sullivan 2016, S. 6). »Liste als Anti-Erzählung« schließlich heißt ein Kapitel aus Ann Cottens Buchs Nach der Welt über Die Listen der Konkreten Poesie und ihre Folgen (2008).
} 
erzähltheoretischer Ansätze. ${ }^{4}$ Als alternative, wenn nicht gegensätzliche Sinnorganisationsform verzichten Listen auf die sinnstiftenden Zeit-, Handlungs- und Motivationsstrukturen von Erzählungen, mit der eine Kette von Ereignissen, Handlungssequenzen, die Konstellation von Figuren sowie räumliche und zeitliche Verhältnisse narrativ geordnet werden. Während in Erzähltexten außerdem eine mehr oder minder manifeste Erzählinstanz vorhanden ist (vgl. Schmid 2008, S. 78-82), die als wenn auch nur imaginierte - »Stimme« den Ausgangspunkt oder die »Ich-Origo « des Erzählens darstellt, fällt die Antwort auf die Frage »Wer spricht?« im Fall von Listen intrikat aus, da »es [häufig] keine >Stimme< gibt, die irgendetwas >sagt«« (Cotten 2008, S. 11).

Listen unterscheiden sich von Erzählungen also erstens durch die relative An- beziehungsweise Abwesenheit von »indizialen Zeichen« (Schmid 2008, S. 79), die auf eine erzählende/auflistende Instanz verweisen, zweitens durch die unterschiedlichen temporalen oder motivationalen Verknüpfungsmodalitäten zwischen den einzelnen Elementen/Ereignissen und drittens durch die unterschiedlichen Grade von relativer Geschlossenheit und Totalität auf der einen, Offenheit und Partikularität auf der anderen Seite.

Wenn man Listen so wie Narrative als Medien kultureller Selbstverständigung und gesellschaftlicher Selbstbeschreibung versteht, die sie zweifellos sind, dann lassen sich aus den genannten formalen Differenzen zwischen Listen und Narrativen Schlussfolgerungen bezüglich der je unterschiedlichen sozialen Effekte der beiden Repräsentationsformen ableiten. Listen und Narrative unterscheiden sich etwa durch das $\mathrm{Maß}$ an Auktorialität und Zurechenbarkeit, über das sie jeweils verfügen, sowie durch die Kausalitäts- und Zeitstrukturen, die Erzählungen inhärent, Listen hingegen äußerlich sind. Ebenso unterscheiden sie sich in der Art und Weise, in der sie an der Aushandlung politischer Entscheidungen und sozialer Ordnungen teilhaben. Während Erzählungen derartige Aushandlungsprozesse oder etwa Konflikte durch die Benennung von Handelnden und Handlungszusammenhängen der Beobachtung aussetzen, werden Aushandlungen in Listen allenfalls implizit mitvollzogen, meistens jedoch vorausgesetzt. Listen steuern politische Prozesse, üben juristische Macht aus und regulieren bürokratische Prozesse, während die sie legitimierenden Verfahren meistens uneinsichtig bleiben (vgl. de Goede/Leander/Sullivan 2016). Darüber, durch welche Verfahren die Liste der sicheren Herkunftsländer oder wie die disposition matrix der US-Regierung zustande kommen, geben die Listen selbst keine Auskunft. $^{5}$

Erzählungen also modellieren die zeitliche Ordnung, handeln Begründungen aus, entfalten Figurenkonstellationen, tarieren Differenzen wie die von Fiktionalität und Faktualität, Recht und Unrecht, Macht und Ohnmacht etc. aus; und während sie das alles leisten, setzen sie sich selbst zudem metanarrativen Reflexionen aus. Listen hingegen nivellieren die Bedeutung der Zeitdimension, ordnen ihre Elemente nicht kausal oder temporal, sondern nominal, ordinal oder metrisch an. Außerdem sind

\footnotetext{
4 »Ein Text ist genau dann eine Erzählung, wenn er von mindestens zwei Ereignissen handelt, die temporal geordnet sowie in mindestens einer weiteren sinnhaften Weise miteinander verknüpft sind.«(Köppe/Kindt 2014, S. 43).

${ }^{5}$ Zur \disposition matrix «, »the US government’s main kill list«, vgl. Weber (2016).
} 
sie im Hinblick auf ihre Ordnung nicht rechenschaftspflichtig, weil die Dinge oder Wörter auf der Liste dadurch, dass sie auf der Liste stehen, eine nicht weiter zu begründende Äquivalenzklasse bilden. Während Erzählungen nach dem Prinzip der Kontiguität aufgebaut und syntagmatisch zu lesen sind, bilden Listen ein Paradigma nach dem Prinzip der Äquivalenz.

\section{Das Paradigma der Liste}

Die Formkonstanz der Liste durch die Geschichte der Schrift hindurch ist verblüffend. Trotz ihrer funktionalen Vielfalt gilt: Listen sind immer Listen, und sie sind immer als solche erkennbar. Gegenüber dem Wiedererkennungswert von Listen erscheint die Bestimmung der generischen Einheitlichkeit von etwa lyrischen Texten ungleich komplizierter. Aber wie genau lässt sich die formale Konstanz von Listen auf den Begriff bringen? Was sind Listen?

Die Antwort lautet recht schlicht und einfach: Die gemeinsame Eigenschaft, die alle Listen auszeichnet, ist ihre Paradigmatik. Listen sind >Paradigmakonstrukte< (Cotten 2008, S. 23). Auch Moritz Baßler definiert Listen als Paradigmen und identifiziert diese »als Herzstück aller popliterarischen Verfahren zwischen Sammeln und Generieren « (Baßler 2005, S. 102). Bei Baßler ist die Liste bereits als literarisch-ästhetisches Verfahren gedacht (dazu später mehr), zunächst aber wäre Folgendes zu klären: Der Begriff des Paradigmas, der hier ins Spiel kommt, geht zurück auf Roman Jakobson, der in einem wegweisenden Aufsatz über »Linguistik und Poetik« die Genese von Texten durch das Zusammenspiel von Selektions- und Kombinationsprozessen beschreibt (vgl. Jakobson 1979). Jakobsons Achsenmodell der Sprache unterscheidet zwischen der vertikalen, paradigmatischen Achse der Selektion, auf der die sprachlichen Elemente im Verhältnis der Äquivalenz zueinander stehen, und der horizontalen, syntagmatischen Achse der Kombination, deren Elemente Kontiguitätsbeziehungen aufbauen. Während die Elemente des Paradigmas eine Äquivalenzklasse bilden, die zum Beispiel grammatisch, phonologische oder semantisch konfiguriert sein kann, folgt das Syntagma syntaktischen Regeln der Kombination.

Demnach stehen die auf einer Liste versammelten Elemente im Verhältnis der Äquivalenz zueinander. Sie bilden ein gemeinsames Paradigma. Um einige Beispiele zu nennen: Auf einer Liste stehen so unterschiedliche Dinge wie Namen (Gästeliste), Telefonnummern (Telefonbuch), Produkte aus dem Supermarkt (Einkaufsliste), Ausflugsziele (Reiseführer) oder Hotels (tripadvisor) ${ }^{6}$, Bücher (Bestsellerlisten), Songs (Charts), Staatsfeinde und Terroristen (kill list) etc. Das inhaltliche Spektrum von Listen ist damit längst nicht erschöpft. Die Geschichte eines Jahres, Jahrzehnts, Jahrhunderts usw. lässt sich übersichtlich rekonstruieren, indem man die wichtigsten historischen Ereignisse auflistet. Die Darstellungsweise der »X« »Superlative« ist so verbreitet und beliebt, weil sie es ermöglicht, Gegebenheiten miteinander zu verknüpfen, die nicht viel mehr gemeinsam haben, als eine »gewisse Ähnlichkeit«. In sozialen Medien wie Facebook oder Twitter erscheinen die Einträge zeitlich oder

\footnotetext{
${ }^{6}$ Vgl. den Beitrag von Urs Stäheli in diesem Heft.
} 
nach Popularität geordnet als Liste. Suchmaschinen präsentieren die Suchergebnisse als Liste. Trotz der funktionalen und typologischen Vielfalt ist allen Listen eines gemeinsam, nämlich dass die Elemente, die auf einer Liste stehen, in eine Beziehung der Äquivalenz zueinander eintreten, dass sie ein Paradigma bilden. Nur durch diese »Beziehung minimaler Äquivalenz« (Stäheli 2012a, S. 92) wird die Liste überhaupt zur Liste. Durch den Eintrag auf der Liste werden die Elemente zu Bestandteilen dieser oder jener Liste vereinheitlicht, ganz gleich wie heterogen die einzelnen Elemente der Liste auf den zweiten Blick sein mögen, welche Funktion sie konkret erfüllen oder ob das Paradigma selbstzerstörerisch durchbrochen wird (vgl. Cotten 2008, S. 24).

\section{4 »alle waren da:«}

Nun: Was bringt es, Listen als Paradigmen zu definieren? Was folgt aus der Unterscheidung zwischen der Struktur der Liste und des Narrativs? Welche Bedeutung kommt der Kontingenz der Liste zu? Dies lässt sich am besten an einem literarischen Beispiel veranschaulichen, das es zugleich ermöglicht, die ästhetische und soziale Dimension von Listen zu fokussieren. Das Beispiel stammt aus dem Buch Angela Davis löscht ihre Website. Listen, Refrains, Abbildungen von Andreas Neumeister aus dem Jahr 2002. Dieses Buch bezeichnet sich als zeithistorisches Dokument, in dem »das Zeitalter, in dem wir leben/die Epoche, in der wir leben« (Neumeister 2002, S. 45), mithin die Gegenwart festgehalten wird. In Neumeisters Buch findet sich unter anderem die folgende Liste:

alle waren da:

Monti Lüftner war da

Ralph Siegel war da

Katja Kessler war da

Franz Josef Wagner war da

Ariane Sommer war da

Matthias Döpfner war da

Jürgen Drews war da

Dieter Bohlen war da

Ulla Kock am Brink war da

Kai Diekmann war da

Hellmuth Karasek war da

Hansi Hinterseer war da

Karl Moik war da

Nadja ab del Farrag war da

Moritz Hunziger war da

Janina Youssefian war da

Guido Westerwelle war da

Shawne Borer-Fielding war da

Jürgen Möllemann war da

Bettina Röhl war da 
Jenny Elvers war da

Ronald Barnabas Schill war da

André Rieu war da

Stefan Raab war da

Ben Becker war da

Carolin Reiber war da

Patrick Lindner war da

Jeanette Biedermann war da

Heinz Heinrich Bauer war da

Nathalie Weidenfeld war da

alle (ebd., S. 13)

Die Angaben, die diese Liste macht, sind ebenso unbestimmt (Wer ist »alle «?; Wo ist »da «?) wie konkret. Die Liste wird von der Bezeichnung einer indefiniten Gesamtheit (»alle«) gerahmt. Wer »alle« sind, wird zwar in der Auflistung näher spezifiziert, die Gemeinsamkeit der genannten 30 Personen wird aber nicht benannt, sondern bleibt offen und muss nachträglich erschlossen werden (was vermutlich mit wachsendem historischen Abstand zunehmend mehr Wikipedia-Recherche erfordert). Was die genannten Leute verbindet, nämlich dass sie zu Beginn der 2000erJahre als Prominente aus Politik, Journalismus, Fernsehen und/oder Musikbranche das soziale Leben der »Berliner Republik « mitbestimmten, die Schlagzeilen der Boulevardblätter füllten und mehr oder minder populär waren, setzt die Liste voraus, beziehungsweise schafft sie dieses Paradigma erst. Die Auflistung von Personen, Phänomenen, Marken, Produkten der Gegenwartskultur betreibt, so Baßler, »kulturelle Poiesis, sie schafft die Paradigmen erst, von denen sie zehrt « und veorrtet die aufgelisteten Elemente auf diese Weise in einem »ästhetisch-popsemiotischen Zusammenhang « (Baßler 2015, S. 115).

Einige der genannten Personen sind immer noch »da«, im Fernsehen (im Dschungelcamp oder bei Promi Big Brother), in der Zeitung, in der Musikbranche, mit ihren Social Media Accounts, andere sind bereits tot und vergessen. Aber auch ohne diese nachträgliche Referenzialisierung der Namen und die Identifikation eines gemeinsamen semantischen Merkmals, stellt die Liste qua Form - und in diesem Fall durch die sprachlichen Wiederholungsstrukturen überbetont - klar, dass zwischen den Namen eine wenn auch zunächst nicht weiter qualifizierte Äquivalenz besteht. Sie baut dadurch eine Isotopie auf.

Die Gemeinsamkeit der genannten Leute besteht ferner in der Tatsache, dass sie »da« waren. Die euphorische Behauptung, »alle« - einfach alle - seien da gewesen, suggeriert zwar eine ausnahmslose, d.h. auch: exklusionslose Allgegenwart der Leute, die »dazu« (Wozu? Zum Boulevard?) gehören. Sie provoziert damit aber erstens die Frage, wer genau denn eigentlich »alle« sind, wer also im Einzelnen da war? Antwort: Eben alle, die man so kennt. Sie provoziert aber zweitens und noch viel dringlicher die Frage danach, wer nicht da war, wer fehlte, »denn der Vorgang [des Auflistens] impliziert, daß einzelne Elemente ein- und andere ausgeschlossen werden« (Goody 2012, S. 384).

Weiterhin bleibt unklar, wo genau sie alle waren, da die Deixis des Lokaladverbiums ohne die Angabe der Sprecherorigo ins Leere läuft. Die Liste ist sowohl 
räumlich als auch zeitlich unbestimmt. Die Liste erzählt (von) nichts. Sie spricht lediglich von der vergangenen Anwesenheit der genannten Medienpersonen. Sie waren da, sind aber schon wieder weg. Während das Lokaladverbium »da« insbesondere durch die epiphorische Repetition die Präsenz der Personen unterstreicht, markiert das ebenso häufig wiederholte Imperfekt von »sein« die Vergangenheit ihrer Gegenwärtigkeit, die gegenwärtige Abwesenheit der genannten Leute. Dabei bleibt die zeitliche Ordnung genauso unbestimmt wie die räumliche. Wann genau und wo die Leute da waren, bleibt eine Leerstelle. Die Liste stiftet weder räumliche noch zeitliche und ebenso wenig motivationale Zusammenhänge, denn daraus, dass Ronald Barnabas Schill da war folgt nicht etwa, dass André Rieu auch da war oder umgekehrt.

»alle waren da:«, um dem Text einen Titel zu geben, den er nicht hat, ist ein typischer popliterarischer Neumeister-Text, der sich dadurch auszeichnet, dass er eine »eigene rhythmische Oberfläche« generiert (Baßler 2005, S. 149) und Formeln und Sentenzen »in rhythmisierte Reihen überführt, die den sequenzierten Stil von Nachrichtentickermeldungen an die Stelle von narrativ entfalteten Handlungszusammenhängen setzen« (Schumacher 2003, S. 47). Popliterarisch ist die Liste, weil sie, mit Moritz Baßler und Eckhard Schumacher gesprochen, durch ihre sprachliche Form rhythmische Oberfläche und nicht-narrative Serialität zum ästhetischen Prinzip macht. »Das Wort Pop setzt auf Wiederholung. Und Klang«, schreibt Neumeister in seinem Manifest über »Pop als Wille und Vorstellung « (Neumeister 2013, S. 269) Dies gilt nicht nur für das Wort Pop, sondern genauso für die popliterarischen Artefakte, etwa diese Liste. Dadurch zeichnet sie sich als poetische Liste aus, »bei der es um die Klänge geht, um die lautliche Gestalt der Liste, das heißt um Signifikanten « (Eco 2009, S. 118), während praktische Listen eine »rein referentielle Funktion« haben (ebd., S. 113). »Der Inhalt einer Liste wird sekundär, der Sinn tritt hinter den Klang oder die Erscheinungsform zurück, also hinter den Rhythmus.« (Cotten 2008, S. 185)

Hört man genau hin, zeichnen sich die auf Neumeisters Liste versammelten Namen in den meisten Fällen besonders durch ihren Klang oder ihren Rhythmus aus, durch die ihnen eigene poetische Funktion im Sinne Jakobsons. Dabei handelt es sich vor allem um phonologische Äquivalenzen wie z.B. die Alliterationen bei Katja Kessler, Hansi Hinterseer oder Ben Becker oder um metrische Äquivalenzen wie bei den Trochäen in Ulla Kock am Brink oder Nadja ab del Farrag. ${ }^{7}$

Es ist daher schwer zu sagen, ob überhaupt ihre Bekanntheit oder Popularität den versammelten Personen Zugang zu Neumeisters Liste verschafft hat oder ob es die poetische Tauglichkeit ihrer Eigennamen war. Ihre »rein referentielle Funktion « hat diese Liste jedenfalls längst eingebüßt, entweder nach ihrer eventuellen Benutzung als Gästeliste für ein Berliner Boulevard-Event der frühen 2000er-Jahre oder spätestens nach der Berichterstattung über dieses Event in Bunte oder Gala. Dieser Verlust von Fremdreferenz betrifft Neumeisters Liste ebenso sehr wie Peter Handkes berühmt gewordene »Aufstellung des 1. FC Nürnberg vom 27.1.1968« (Handke 1969, S. 59), die nach der Ankündigung der Aufstellung in der Morgenzeitung oder dem

\footnotetext{
7 Vgl. für ein popliterarisches Beispiel aus der angelsächsischen Literatur die Gästeliste von Victor Ward in Ellis (1999, S. 78-83).
} 
Spielbericht am Tag danach kaum noch Informationswert besitzt. Der Informationsgehalt verlagert sich unterdessen komplett auf die Seite der literarischen Mitteilung, also auf die Information, dass dieser Text als literarischer Text »mitgeteilt« wird. Die fremdreferenzielle Funktion dieser Texte wird zugunsten ihrer Selbstreferenz gekappt, um schließlich ihr eigenes Verfasst- oder Gemachtsein als Text, Liste, Literatur oder Kunst selbstreflexiv zu thematisieren (vgl. Geulen/Geimer 2015, S. 522) und damit den Punkt zu treffen, an dem Auflistung zur Kunst wird (vgl. Mainberger 2003).

\section{Die Ästhetik der Liste}

Listen sind weder schön noch erhaben, sondern interessant (im Sinne von Ngai 2012). Die Alltäglichkeit der Kulturtechnik »Liste« - alle fertigen jeden Tag alle möglichen Listen an - sorgt für ästhetische Irritationen. ${ }^{8}$ Mehr noch: Listen stehen unter ästhetischem Rechtfertigungszwang. Auflisten kann jeder. Darum sind Listen ästhetisch rechenschaftspflichtig, weil sie die Frage nach ihrer künstlerischen Legitimität aufwerfen (vgl. ebd., S. 168f.).

Etwas interessant zu finden und ihm Beachtung zu schenken, markiert zunächst eine Differenz zu den weniger interessanten Gegenständen. Das Urteil, das lediglich eine nicht weiter spezifizierte Differenz (»unqualified difference «) markiert, bringt ein »feeling of not-yet-knowing « zum Ausdruck (ebd., S. 132). Dieses unbestimmte Interessant-Finden fungiert in der Regel als Aufforderung, das ästhetische Urteil näher zu begründen beziehungsweise zu rechtfertigen. »[T]he deepest content of the aesthetic category of the interesting is precisely that of the justification of aesthetic judgements in general.« (ebd., S. 170)

Weil Listen und Listenstrukturen eigentlich langweilig und redundant sind, erregen sie ästhetisches Interesse, das rechtfertigungsbedürftig ist. Neumeisters Liste etwa ist aus mehreren Gründen interessant: Es ist interessant zu wissen, wer alles da war. Insofern die Liste Auskunft über die Anwesenheit gewisser Leute bei einem bestimmten Anlass gibt, besitzt sie einen Informationswert, der jedoch durch ihre Unbestimmtheit, das zentrale Moment des Interessanten (vgl. ebd., S. 131), zugleich radikal entwertet wird. Die Liste gibt Informationen, an denen man schon in dem Moment, in dem man sie erhält, das Interesse verliert. Innerhalb der Liste wiederholt sich diese Informationsentwertungslogik. Die und der waren auch da. Aha, interessant! Mehr nicht. Das veranschaulicht in etwa, was Ngai darunter versteht, dass die ästhetische Kategorie des Interessanten eine Reflexionsform für die Zirkulation von Informationen in der spätkapitalistischen Gesellschaft sei (ebd., S. 1f.). Nach der Steigerungslogik des Interessanten, dass es immer noch interessanter geht, ist niemals ein Absolutes wie das Schöne erreicht. Das Neuheits- und Aktualitätsversprechen des Interessanten wird durch das ständige Voranschreiten der Zeit permanent enttäuscht. ${ }^{9}$

\footnotetext{
8 So argumentiert auch Eva von Contzen in ihrem Beitrag zu diesem Heft.

${ }^{9}$ Das ist durchaus im Schlegel'schen Sinne >progressiver Universalpoesie <, die »noch im Werden « ist, zu verstehen. Von Schlegel leitet Ngai die ästhetische Kategorie des Interessanten ab.
} 
Ihre ästhetische Hochzeit erleben »Listen und Listenverwandtes « (Diederichsen 2006, S. 110) in der Popliteratur und in der Konzeptkunst der 1960er-Jahre (vgl. ebd.; Ngai 2012, S. 33, 143f.). Hubert Fichtes »Palettenabc«, das in alphabetischer Reihenfolge auflistet, was typisch für das titelgebende Szenelokal ist, von Adenauer, Anchovis und Arsch bis Zocken, erscheint 1968 (Fichte 2010, S. 153-156, 187-190). Handkes sprachexperimenteller Band Die Innenwelt der Außenwelt der Innenwelt, der den Weltkontakt von Sprache und Literatur problematisiert und der das ready made-Gedicht mit der Fußballaufstellung enthält, erscheint 1969. Für diese Blütezeit des Auflistens (vgl. Hecken/Werber 2017) finden sich verschiedene Begründungen: die »Orientierung an Alltagssprache und Alltagstext«; eine »Fortsetzung oder Rückkehr jener unmittelbaren und bevorzugt als indexikal ausgewiesenen Bezugnahme auf >Realität « «, die »readymademäßig « ausgestellt werden soll (Diederichsen 2006, S. 113); das Interesse der indexbasierten, nachpopulären Künste an einem »Realismus [...], der die Narration durchbricht« (vgl. Diederichsen 2017, S. 71); »stylistic pluralism and hybridity, and the seemingly endless pursuit, in the felt absence of any totalizing vision « (Ngai 2012, S. 146). Die paradigmatische Äquivalenzstruktur der Liste sieht keine Metaposition vor und veranschaulicht auf diese Weise, dass es keinen Überblick über >das Ganze < und keine »totalizing vision « gibt. ${ }^{10}$

\section{Populärkultur}

Besonders bemerkenswert für die interessante Ästhetik der Liste erscheint uns jedoch die Bedeutung der Populärkultur zu sein, wie sie sich seit den 1950er-Jahren herausbildet, und zwar insbesondere die Art und Weise, wie populärkulturelle Bestände seitdem sortiert und präsentiert werden: nämlich als Liste. Das Wissen der Populärkultur insgesamt ist in Listen organisiert und geordnet, seitdem der Begriff des Populären im Laufe der 1950er und 1960er Jahre eine Transformation von einem normativen zu einem quantitativen Verständnis erfahren hat (vgl. Schaffrick 2016). Das lässt sich insbesondere an der wachsenden Bedeutung von Charts und Bestsellerlisten ablesen. Um nur ein Beispiel dafür zu nennen: Nachdem die von der Literarischen Welt 1927 ins Leben gerufene erste deutsche Bestsellerliste bereits 1929 wieder eingestellt wird, lebt diese Bewertungspraktik 1957 mit dem »SellerTeller« in der Zeit wieder auf, um schließlich mit der Bestsellerliste des Spiegel eine bis heute einflussreiche und populäre Institution auszubilden (vgl. Schaffrick 2018).

Wie auch Charts tragen Bestsellerlisten dazu bei, dass der »Erfolg und die Popularität von Musikstücken und Künstlern«, aber auch von Büchern, Filmen, Eissorten, Hotels, Universitäten etc. »v.a. auf quantitativen Kriterien« basieren (NeumannBraun/Schmidt 2003, S. 133). Dies erfordert eine Definition des Populären, die populär nicht als das konzeptualisiert, was >einfach<, >eingängig<, > ursprünglich < oder >volkstümlich< ist, sondern dem Populären eine quantitative Bedeutung gibt: »Populär ist, was viele beachten« (Hecken 2006, S. 85). Unter dem Einfluss popästhetischer

10 Die »felt absence of any totalizing vision« diskutieren Johannes Paßmann und Carolin Gerlitz in ihrem Beitrag zu diesem Heft am Beispiel des Knolling, das sich passenderweise zu Fotomotiven der 1960erJahre (und noch weiter) zurückverfolgen lässt. 
Innovationen wie der Inklusion kulturindustriell gefertigter Produkte in den Bereich der Kunst überwindet auch das Populäre selbst die Differenz von >hoher $<$ und $>$ niederer $<$ Kunst, high und low. Diese seit Kant und Schiller gepflegte Unterscheidung wird seit den 1950er-Jahren allmählich durch die quantitative Ordnung des Populären in Ranglisten ersetzt. Listen wie Ratings und Rankings avancieren zur Bedingung der Möglichkeit für die Konstitution, Kommunikation und Evaluation des Populären. Bei den Listen der Populärkultur handelt es sich also wie bei Charts, Bestsellerlisten, Rankings (Ranglisten) etc. entweder um ordinale, d.h. hierarchisch sortierte, oder, wie bei Ratings, um metrische Listen, in denen zusätzlich zu der hierarchischen Ordnung die Abstände zwischen den Rängen quantifiziert werden. Diese Listen werden in der Regel unter Einbeziehung möglichst großer Datenmengen elektronisch oder algorithmisch generiert, also durch Programme, die von dem, was sie prozessieren, nicht das Geringste verstehen: organizing without understanding. ${ }^{11}$ In den Listen der Populärkultur werden Publikumsresonanz und Popularität nach quantitativen Kriterien erfasst und inszeniert, aber auch überhaupt erst in kommunizierbarer Form konstituiert und schließlich in Konsumempfehlungen transformiert. Ranglisten und Rankings selbst sind als Agenten an der Popularisierung der quantitativen Vorstellung von Popularität beteiligt, auch weil nur das, was quantifizierbar ist, überhaupt als Eintrag auf diesen Listen erscheint und damit Beachtung und Anerkennung findet (vgl. Werber 2017).

Dabei handelt es sich um praktische Listen, die am Ursprung von Handlungen stehen. Diese Handlungen allerdings sind keineswegs vorhersehbar, sondern vollkommen unbestimmt und »stets verlagert, verschoben, dislokal« (Latour 2010, S. 82). Die Listen versammeln und verwalten die Ergebnisse von Handlungen wie Kaufentscheidungen oder Wahlen ebenso wie sie umgekehrt Handlungen initiieren. Indem sie Handlungsoptionen - Hotelbuchung, Lektüre, Playlists etc. - benennen, hierarchisieren, gewichten, regulieren und verwalten, tragen sie zur Ausbildung von sozialen Akteuren bei. Listen sind also »Mittler« im Sinne Bruno Latours, d.h. sie »übersetzen, entstellen, modifizieren und transformieren die Bedeutung oder die Elemente, die sie übermitteln sollen« (ebd., S. 70), indem die Listen sie mit einem Popularitätsindex versehen und sie in das Paradigma des Populären integrieren.

\section{Flache Vergangenheit}

Die nominalen Listen der Popliteratur und Popkultur unterscheiden sich grundlegend von den quantitativen Listen der Populärkultur (vgl. Schaffrick 2016). Ihre Funktion besteht nicht darin, Quantitäten zu verwalten und zu gewichten. Neumeisters »alle waren da:« gibt keine Auskunft darüber, wer der Populärste unter den Prominenten, wer A-, B- oder C-Promi ist. Stattdessen reflektieren die popkulturellen Listen die Effekte der populärkulturellen Praktiken des Auflistens. Zieht man noch einmal Neumeisters Liste als Beispiel heran, so zeigt sich, dass »das Zeitalter, in dem wir leben/die Epoche, in der wir leben« (Neumeister 2002, S. 45) kein Zeitalter und

11 So lautet der Titel von Elena Espositos Beitrag zu diesem Heft, in dem sie sich mit der Unterscheidung von Ratings und Rankings und der algorithmischen Produktion von Listen befasst. 
keine Epoche der Narrative ist, sondern der Liste. Neumeisters Liste erzählt (von) nichts und zählt nicht auf, und dennoch oder gerade deshalb lassen sich aus ihr Rückschlüsse auf die populärkulturelle Konstitution des Sozialen ziehen.

»Wenn wir keine Geschichten über die Gesellschaft erzählen können, dann müssen wir Listen erstellen.« (Stäheli 2012a, S. 83) Die eingangs eingeführte Differenz von Narrativ und Liste kehrt hier in ihrer soziologischen Dimension mit all ihren Konsequenzen für die Konstitution des Wissens der Gesellschaft über sich selbst zurück. Wie sähe die Geschichte zu Neumeisters Liste aus? Was spielte sich ab auf dem roten Teppich, an der Bar, auf der Tanzfläche? Wer wurde mit wem gesehen? Und warum verließ wer wann die Veranstaltung? Die Geschichte wäre lang und kompliziert. Insbesondere die Frage nach der Begründung des Sozialen, nach der Gesamtheit, dem Ganzen der Gesellschaft, nach Inklusion und Exklusion stünde zur Disposition. Wer sind »alle«? Warum war die eine da, der andere nicht? Narrative können darauf multiperspektivisch vielstimmige Antworten geben, je nachdem, aus wessen Perspektive die Geschichte erzählt wird. Über diese Möglichkeiten verfügen Listen nicht. So ist Neumeisters Liste also einfach eine Liste, paradigmatisch.

Besonders sticht bei Neumeister die Auflösung zeitlicher Differenzen hervor. Das Imperfekt »waren « bleibt ohne adverbiale Zeitangabe unbestimmt. Welche Vergangenheit ist damit gemeint? Hinzu kommt, dass die Liste nun, also mit unbestimmter zeitlicher Distanz, Personen versammelt, die entweder immer noch medial präsent oder aber bereits tot sind. Ihr Nebeneinander auf der Liste enthebt sie der Vergänglichkeit. Die Vergangenheit wird flach.

Während die Zeitdimension für die Sinnkonstitution von Gesellschaft unerlässlich ist und meist narrativ gehandhabt wird, lösen Listen Zeitordnungen auf. Da Listen generell über keinen Zeitindex verfügen, ist der von Neumeisters Liste inszenierte Effekt etwas, das sich verallgemeinern lässt. In den Musik-Charts z.B. können unterschiedslos Songs oder Alben aus jeder Phase der Musikgeschichte stehen, ohne dass zeitliche Differenzen markiert würden. Immer wieder steht das zeitlose »Last Christmas« ganz oben in den Popularitätsrankings der Weihnachtszeit, die klassischen Komponisten werden neu eingespielt, die Beatles remastered, und alle diese Musikstücke stehen, wenn die Verkaufszahlen es wollen, direkt neben Lana Del Ray und Lady Gaga. Auf historische Abstände, stilgeschichtliche Differenzen und chronologische Abfolge kommt es nicht an. »alle waren da:«, und alle sind gleich weit von der Gegenwart entfernt, zeitlich äquivalent. Nicht nur das Mittelalter, sondern alle denkbaren Vergangenheiten dienen in der Populärkultur als »heterogener Fundus, in dem alles je gleich weit entfernt ist und miteinander zu verschiedenen Zwecken kombiniert werden kann « (Groebner 2008, S. 136). Die listeninduzierte Zeitdiffusion führt zu einer flachen Vergangenheit, die im Gegensatz zum Chronotopos der >breiten Gegenwart< steht (Gumbrecht 2010). Denn weder wird die Gegenwart von ständig präsenten Vergangenheiten >überschwemmt<, die nicht vergessen werden können (ebd., S. 16), noch stellen sich dabei Präsenzeffekte ein. »alle waren da:«, aber »alle« sind nicht alle, die möglicherweise hätten da sein können und auf der Liste vergessen wurden. Und »alle« sind nicht mehr präsent, sondern ihre Präsenz ist bereits vergangene Gegenwärtigkeit. Ansonsten lässt die Liste vieles unbestimmt. So viel Unbestimmtheit können Listen sich erlauben, und das macht sie nicht nur ästhetisch interessant, sondern auch wissenschaftlich relevant. 


\section{Literatur}

Baßler, Moritz: Der deutsche Pop-Roman. Die neuen Archivisten. München 2. Aufl. 2005.

Baßler, Moritz: »Definitely Maybe. Das Pop-Paradigma in der Literatur«. In: Pop. Kultur und Kritik 4 (2015), Heft 6, S. 104-127.

Contzen, Eva von: »The Limits of Narration. Lists and Literary History«. In: Style 50 (2016), Heft 3, S. 241-260.

Cotten, Ann: Nach der Welt. Die Listen der Konkreten Poesie und ihre Folgen. Wien 2008.

de Goede, Marieke/Leander, Anna/Sullivan, Gavin: »Introduction: The politics of the list «. In: Environment and Planning D: Society and Space 34 (2016), Heft 1, S. 3-13.

Diederichsen, Diedrich: Körpertreffer. Zur Ästhetik der nachpopulären Künste. Berlin 2017.

Diederichsen, Diedrich: »Liste und Intensität«. In: Dirck Linck/Gert Mattenklott (Hg.): Abfälle. Stoffund Materialpräsentation in der deutschen Pop-Literatur der 60er-Jahre. Hannover-Laatzen 2006, S. 107-123.

Eco, Umberto: Die unendliche Liste. München 2009.

Ellis, Bret Easton: Glamorama. New York 1999.

Fichte, Hubert: Die Palette. Roman [1968]. Frankfurt a.M. 2. Aufl. 2010.

Geulen, Eva/Geimer, Peter: »Was leistet Selbstreflexivität in Kunst, Literatur und ihren Wissenschaften?« In: DVjs 89 (2015), Heft 4, S. 521-533.

Goody, Jack: »Woraus besteht eine Liste?« In: Sandro Zanetti (Hg.): Schreiben als Kulturtechnik. Grundlagentexte. Berlin 2012, S. 338-396.

Groebner, Valentin: Das Mittelalter hört nicht auf. Über historisches Erzählen. München 2008.

Gumbrecht, Hans Ulrich: Unsere breite Gegenwart. Berlin 2010.

Handke, Peter: Die Innenwelt der Außenwelt der Innenwelt. Frankfurt a.M. 1969.

Hecken, Thomas/Werber, Niels: »Literatur«. In: Thomas Hecken/Marcus S. Kleiner (Hg.): Handbuch Popkultur. Stuttgart 2017, S. 178-188.

Hecken, Thomas: Populäre Kultur. Mit einem Anhang >Girl und Popkultur<. Bochum 2006.

Jakobson, Roman: »Linguistik und Poetik « [1960]. In: Elmar Holenstein/Tarcisius Schelbert (Hg.): Poetik. Ausgewählte Aufsätze 1921-1971. Frankfurt a.M. 1979, S. 83-121.

Köppe, Tilmann/Kindt, Tom: Erzähltheorie. Eine Einführung. Stuttgart 2014.

Koschorke, Albrecht: Wahrheit und Erfindung. Grundzüge einer Allgemeinen Erzähltheorie. Frankfurt a.M. 2012.

Koschorke, Albrecht: »Codes und Narrative. Überlegungen zur Poetik der funktionalen Differenzierung«. In: Walter Erhart (Hg.): Grenzen der Germanistik. Rephilologisierung oder Erweiterung? Stuttgart/ Weimar 2004, S. 174-185.

Latour, Bruno: Eine neue Soziologie für eine neue Gesellschaft. Einführung in die Akteur-Netzwerk-Theorie. Frankfurt a.M. 2010.

Mainberger, Sabine: Die Kunst des Aufzählens. Elemente zu einer Poetik des Enumerativen. Berlin/New York 2003.

Martínez, Matías/Scheffel, Michael: Einführung in die Erzähltheorie. München 9., erw. u. akt. Aufl. 2012.

Meuter, Norbert: »Geschichten erzählen, Geschichten analysieren: Das narrativistische Paradigma in den Kulturwissenschaften«. In: Friedrich Jaeger/Jürgen Straub (Hg.): Handbuch der Kulturwissenschaften. Bd. 2: Paradigmen und Disziplinen. Stuttgart/Weimar 2011, S. 140-155.

Müller-Funk, Wolfgang: Die Kultur und ihre Narrative. Eine Einführung. Wien/New York 2. Aufl. 2008.

Neumann-Braun, Klaus/Schmidt, Axel: »Charts«. In: Hans-Otto Hügel (Hg.): Handbuch Populäre Kultur. Begriffe, Theorien und Diskussionen. Stuttgart/Weimar 2003, S. 131-134.

Neumeister, Andreas: Angela Davis löscht ihre Website. Listen, Refrains, Abbildungen. Frankfurt a.M. 2002.

Neumeister, Andreas: »Pop als Wille und Vorstellung«. In: Charis Goer/Stefan Greif/Christoph Jacke (Hg.): Texte zur Theorie des Pop. Stuttgart 2013, S. 262-269.

Ngai, Sianne: Our Aesthetic Categories. Zany, Cute, Interesting. Cambrigde (MA)/London 2012.

Nünning, Ansgar: »Wie Erzählungen Kulturen erzeugen: Prämissen, Konzepte und Perspektiven für eine kulturwissenschaftliche Narratologie«. In: Alexandra Strohmaier (Hg.): Kultur - Wissen - Narration. Perspektiven transdisziplinärer Erzählforschung für die Kulturwissenschaften. Bielefeld 2013, S. $15-53$.

Schaffrick, Matthias: »Listen als populäre Paradigmen. Zur Unterscheidung von Pop und Populärkultur«. In: KulturPoetik 16 (2016), Heft 1, S. 109-125. 
Schaffrick, Matthias: »Paratext Bestsellerliste. Zur relationalen Dynamik von Popularität und Autorisierung.« Erscheint in: Martin Gerstenbräun/Nadja Reinhard (Hg.): Paratextuelle Politik und Praxis. Interdependenzen von Werk und Autorschaft. Wien u.a. 2018.

Schmid, Wolf: Elemente der Narratologie. Berlin/New York 2., verbesserte Aufl. 2008.

Schumacher, Eckhard: Gerade Eben Jetzt. Schreibweisen der Gegenwart. Frankfurt a.M. 2003.

Stäheli, Urs: »Listing the global: dis/connectivity beyond representation?«. In: Distinktion: Scandinavian Journal of Social Theory 13 (2012b), Heft 3, S. 233-246.

Stäheli, Urs: »Das Soziale als Liste. Zur Epistemologie der ANT«. In: Friedrich Balke/Maria Muhle/ Antonia von Schöning (Hg.): Die Wiederkehr der Dinge. Berlin 2012a, S. 83-101.

Weber, Jutta: »Keep adding. On kill lists, drone warfare and the politics of databases«. In: Environment and Planning D: Society and Space 34 (2016), Heft 1, S. 107-125.

Werber, Niels: »Willkommen in der Numerokratie«. In: Frankfurter Allgemeine Zeitung (11.07.2017), S. 9. 